Blekinge Institute of Technology Research Report No 2002:08

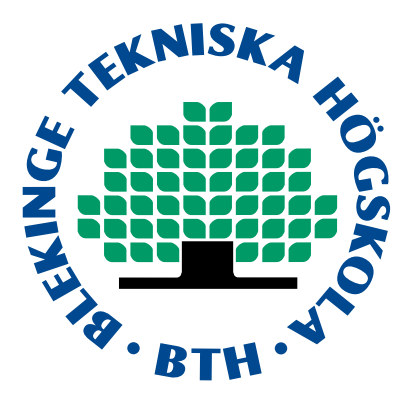

\title{
Modeling and Evaluation of Internet Applications
}

\author{
by \\ Ajik K. Jena, Adrian Popescu and \\ Arne A. Nilsson
}

Department of Telecommunications and Signal Processing Blekinge Institute of Technology 


\title{
Modeling and Evaluation of Internet Applications
}

\author{
Ajit K. Jena \\ Computer Center \\ Indian Institute of Technology \\ Bombay-400076, India \\ ajit@cc.iitb.ac.in
}

\author{
Adrian Popescu, Arne A. Nilsson \\ Dept. of Telecommunications and Signal Processing \\ Blekinge Institute of Technology \\ 37179 Karlskrona, Sweden \\ apo@bth.se,ano@bth.se
}

\begin{abstract}
The paper presents a modeling and evaluation study of the characteristics of several "classical" Internet applications (SMTP, HTTP and FTP) in terms of user behavior, nature of contents transferred and application layer protocol exchanges. Results are reported on measuring, modeling and analysis of application level traces collected, at both the client and the server end, from different environments such as university networks and commercial Frame Relay networks. The methodologies used for capturing traffic flows as well as for modeling are reported. Statistical models have been developed for diverse parameters of applications (e.g., HTTP document sizes, FTP file sizes, and SMTP message sizes), which can be useful for building synthetic workloads for simulation and benchmarking purposes. All three applications possess a session oriented structure. Within each session, a number of transactions are performed. For the above mentioned applications, the number of transactions that may occur during a session has also been modeled.
\end{abstract}

\section{Introduction}

The global Internet has seen tremendous growth in terms of nodes and user base as well as of types of applications. One of the most important consequences of this growth is related to an increased complexity of the traffic experienced in these networks. In order to support Internet applications effectively, it is therefore important to understand and to characterize the application level transactions as well as the effect of different TCP/IP control mechanisms on application-level parameters.

Internet applications like SMTP, HTTP and FTP have been in use for quite some time. Especially the popularity of WWW technology has had a major impact on modern life and has influenced our lives in many ways. It has, for instance, acted as a catalyst in the synergy of traditional telephony and data communication technologies. Furthermore, the fact that the WWW technology is available on demand made it very appealing to the user community. The Web technology has been quickly adapted for specific purposes like education, entertainment, and commercial environments and has become the preferred way of content distribution. As a consequence, researchers have been quite active and tried to understand the complexity of WWW, to develop appropriate workload models for simulation and testbed purposes as well as to improve the performance. The problem is further complicated because the Web is actually consisting of a variety of different software components (e.g., browsers, servers, proxies, back-end databases), and also due to the ever-changing characteristics of Internet traffic.

Today, some of the major challenges in WWW performance are scalability, latency, bandwidth and the problem of aborted connections. Related to this, it is therefore important to understand and characterize the application level characteristics. For instance, significant contributions have been made so far towards characterizing the application (e.g., client behavior, server behavior, proxy behavior and structure of Web pages) $[1,3,14,16]$, towards better protocols $[10,14]$ as well as for better caching strategies for clients and for servers [5, 14]. All these studies have revealed the complexity of WWW and it is observed that fundamental to all studies is the need for a deep understanding of WWW workloads.

Applications like SMTP and FTP have also been extensively studied. For instance, empirical models are reported in [8], whereas other studies [19,25] report detailed models of SMTP and/or FTP connections, together with discussion of heavy-tailed properties.

Finally, another important aspect is related to the development of generators of synthetic workloads, which has been a major focus of Internet research. Accordingly, a number of tools have been reported so far, e.g., [2, 8].

The purpose of the paper is to present a characterization study of traffic collected at clients and servers for the considered applications, to be further used in a client-server simulation framework [12]. The simulation model 
is driven by the above-mentioned applications.

Detailed results are reported on measuring, modeling and analysis of traffic collected from different (classes of) sites together with methodologies used for capturing traffic flows as well as for modeling. In particular, we confirm results of former studies showing that the distribution of HTTP document sizes can be modeled by a mixture of Lognormal and Pareto distributions as well as that diverse classes of Web servers (e.g., academic, entertainment, commercial) seem to show structural similarities in their distributional properties. Furthermore, it is also shown that the number of embedded documents in a Web page can be well modeled by a Negative Binomial distribution and a model is put forth that can be used for the generation of the number of embedded objects in a Web page. In addition, other several properties observed earlier are revealed, which seem to be specific for SMTP and FTP. These are for instance the bimodal structure in the body size distribution for SMTP messages and also the fact that the objects transferred by using FTP are files whose size distributions are likely to be a mixture of Lognormal and Pareto distributions. Finally, an important point in our study is regarding the common modeling structure that is used to model all the three different applications. This takes the form of transaction oriented structure of application sessions, which is useful in developing the simulation model.

The paper is organized as follows. Section 2 describes the measurement infrastructure used for this study. The collection of data traffic is presented in section 3. Section 4 describes the appropriate modeling methodology used. Section 5 presents the SMTP characteristics considered in the study together with the summary statistics for the collected data sets. Section 6 describes the HTTP characteristics that are considered in the study. It also reports the summary statistics for the collected data sets and a model is put forth for the number of embedded documents in a Web page. Finally, section 7 presents the considered FTP characteristics as well as the summary statistics for the collected data sets. The paper is concluded in section 8 .

\section{Measurement Infrastructure}

In order to model the ON-OFF behavior of the application sources, it is essential to collect the protocol message elements along with their respective message timings. Several measurement utilities have been used for doing traffic monitoring and analysis. The NIKSUN NetVCR ${ }^{T M}$ monitoring and analysis system [17] as well as several application specific tools for analysis of data at both client and server sides have been used.

- The NIKSUN NetVCR ${ }^{T M}$ monitoring and analysis system is a non-intrusive system for network monitoring and analysis that is able to collect and storage data from different link equipments. The system can be connected to all the interfaces in a specific local/wide area network (LAN/WAN) and collect all data traffic in the specific LAN or on the probed WAN interfaces. The observed data traffic can be recorded for later reference on a storage device such as hard disk or tape. Individual packets can be automatically classified into link to application layer data sets and automatically analyzed (e.g., Frame Relay, Ethernet, ATM, IP, TCP, UDP, WWW, MBone). NetVCR's application programming interface (API) allows for retrieving of stored data traffic based on some criteria (e.g., time interval, packet type, IP address, port number).

- The tcptrace utility, for mapping IP packets to TCP flows [18], is used to increase the capabilities of $\mathrm{NetVCR}^{T M}$.

- The augmented tcptrace utility is used to capture the message timings [11].

- The $\boldsymbol{x}$ flowstat utilities, where the term $\boldsymbol{x}$ refers to any of the three applications, i.e., smtp or http or $f t p$, is used to help summarize the huge information generated from the flows and to extract diverse application specific features [11].

- GetWeb mirroring software is used for duplicating the content tree of a designated Web server on the local disks [26], and

- Using of application logs analysis (especially SMTP logs and access logs at FTP servers).

The objectives for the monitoring and analysis infrastructure are to capture the following:

- Type and sizes of application layer objects, for building up session structure models for the three applications.

- Structural similarities and/or differences among characteristics of sessions belonging to different types of applications, and

- Search for possible invariant characteristics in object flows across different user organizations. 


\section{Capturing Application Traffic Flows}

The results reported in the paper are based on datasets with up to about 25000 SMTP flows collected from the teachers' access subnetwork at the Blekinge Institute of Technology (BIT), Karlskrona, Sweden as well as from the Indian Institute of Technology (IIT), Bombay, India. Typically the time periods selected span over a week or 10 days, or sometimes even a month. In fact, the NetVCR ${ }^{T M}$ monitor is continuously attached to the network (Ethernet) and, in order to extract a specific dataset, it is sufficient to specify the application of interest and the period over which the collection is to be done.

It has been observed that about 100 SMTP sessions were usually generated every day, giving a total of about 6000 SMTP sessions for analysis. The message body sizes have been also collected from the SMTP logs of the main mail server at BIT. In addition, the SMTP logs of the mail server at IIT have been collected and used in our analysis as well. Both these hosts handle the bulk of the inter-site email message loads for the respective institutions.

For the HTTP data traffic, we used the NetVCR ${ }^{T M}$ system as well as the Web mirroring software to collect data from a number of sites. The content pages on WWW servers are created by the hosting organizations towards the specific goal of furthering their interests. To that extent, the pages represent the characteristics of the organization. We hypothesized that organizations with similar business have similar content structures and should therefore exhibit some similarities in structure. Accordingly, three classes of servers were identified as possible targets, namely educational, media companies, and companies engaged in information technology business. In each of these categories, three to four different servers were probed. Starting at their homepages, and using a recursive technique, we descended 10 levels of the Web hierarchy and collected their characteristics. This process resulted finally in a collection of 2500 to 10000 observations for each of the servers involved in the experiment.

The other aspects of characterizing the WWW application objects were on the client side. The experiment involves collecting packet traces using NetVCR ${ }^{T M}$ from a user environment. The student and staff access subnetworks at Blekinge Institute of Technology have been used for this experiment. The university user base is quite small and thus the observations generated per day is small. Over measurement periods, a total of about 18000 observations were collected for the staff access subnet and 20000 observations were collected for the student subnet.

Due to the low volume FTP traffic available at the Blekinge Institute of Technology, collection of traffic flows for FTP sessions has been done instead by using access logs at the SUNET FTP server (ftp.sunet.se). This server is placed at the Uppsala University, Sweden, and is one of the major anonymous FTP service providers in Scandinavia. Two traces have been collected from this site. Each of the traces provides access logs for about two hours duration. The first trace has about 5000 sessions and the second trace has about 10000 FTP sessions. Both datasets have only anonymous FTP accesses and the server activity shows only download operations.

Further, we have also collected data traffic from IIT Bombay. The server logs from IIT Bombay were collected over a period of three months, in the form of two separate traces. Given the restricted access to the server, the traces produced were pretty small, having about 100 FTP sessions each. The server activity at IIT Bombay shows both downloads and uploads.

We finally mention that the amount of data traffic collected for these applications, and also the distributional properties derived therefrom, has been proven to be large enough to help us in building a rough simulation model [12].

\section{Modeling Methodology}

Detection and estimation of heavy-tailed properties in the distributions of application layer objects is an important aspect in performance modeling of applications. It may for instance reveal the presence of infinite mean or variance. Accurate estimation of these properties is also important in order to capture the degree of Long-Range Dependence (LRD) inherent in the objects [6]. Such estimates are also very useful in building simulation models that can reproduce traffic conditions as observed in real networks.

Often the random variable possessing heavy tail appears hidden behind another distribution. While the two distributions may have very different tail behavior in a mathematical sense, it may be quite difficult to segregate the two in a practical fitting problem. For instance, it has been observed that the sizes of HTTP objects have usually a distribution that can be described as Lognormal and this can contribute with about 80 to $95 \%$ of the probability mass with the remaining mass contributed by a Pareto distributed random variable [19]. The crux of the problem lies therefore in determining the cutoff point between the two distributions $[6,19]$.

The procedure for fitting probability distributions for mixture models is as follows [13, 19]: 
- The first step is to look at the histogram and empirical distribution plot and the summary of the observations (such as minimum, maximum, mean, median, variance, etc). This step helps in eliminating many candidate distributions. This may also help in determining if the observation data set can be modeled using a single distribution or mixture models are required.

- Visual techniques such as Hill estimator plot [24] and the Complementary Cumulative Distribution Function (CCDF) plot on a log-log scale can be used to inspect whether the set of observations has a heavy tail. The heavy tail shows up as a straight line in the CCDF plot. On the Hill plot, the initial instability quickly dies down and the curve becomes roughly parallel to the x-axis. In the case of mixture models, the parallel behavior is maintained for the entire tail portion. If the distribution of the body portion is Lognormal, then the curve will start rising just after the cutoff point. While it may not be possible to determine the cutoff point accurately, this provides a hint about the tail mass.

- The next step is to try some candidate distributions and perform the null hypothesis test to find out the significance level of the observations having been drawn from the candidate distribution. The AndersonDarling (AD) test provides an adequate framework for this [7]. In the case of mixture model, a method of successive right-censoring is used to partition the observations. The range for the amount of rightcensoring is decided by using the hint from step 2 above. The AD test needs also to be modified to be applicable to censored samples [7, 19]. If the test does not show any significance level when applied to the complete set, the test is applied then to smaller subsamples. In each case 100 tests are performed and the number of times are counted when the $1 \%$ and 5\% significance levels are passed. The null hypothesis is considered to be passed if there are at least 50 trials showing 5\% significance and at least 75 trials showing $1 \%$ significance. If the sample does not meet this significance criteria, then the process is repeated with smaller subsamples. For the HTTP samples, it was observed that the maximum subsample size that met the significance criteria was 1000 and the minimum was 100. In the case of mixture models, a suitable cutoff point was selected where the null hypothesis is best satisfied for both the participating distributions. This was done by using different amounts of censoring and evaluating the significance criteria. In some samples, the significance criteria was very difficult to obtain. In summary, it is true that the null hypothesis test was not passed cleanly and thus the model is not exact in a statistical sense. At the same time, having shown some limited significance levels, it can be stated that the model is close to the specific distribution mixture.

- The parameters for the distributions that passed the null hypothesis in step 3 above are obtained by using the Maximum Likelihood Estimation (MLE) method. Special methods applicable to estimate parameters from censored samples are also used [13].

- Having obtained the distributions and the parameters thereof, a measure of the discrepancy between the observations and the model can be obtained using the $\lambda^{2}$ criteria [19].

\section{SMTP}

The SMTP protocol deals with the electronic mail service on Internet. The users interact with this application by means of the so-called User Agents (UAs), which allow them to compose and send mail messages. UAs support complex encoding and decoding functions for the different body parts. On the receiver side, each user has a separate mailbox that is accessed by the UA to render the message content on the user screen. Further, the exchange of email usig TCP is performed by the Message Transfer Agents (MTAs). The MTA processes the messages arriving from the local users as well as from remote MTAs. It acts as a postal sorting clerk by posting the messages to the local mailboxes or passing the outbound messages to the outbound messages queue. On the transmit side, the MTA takes the messages from the queue and sends them directly to the destination host (or to the authorized host acting on behalf of the destination).

Typically all the components of email system are located within the physical boundaries of a single system but by using the POP or IMAP access protocol the user agents can even be located on a remote host.

In order to exchange emails, the participating hosts must conform to the standards specified in RFC821 [21], which describes how two MTAs communicate with each other across a single TCP connection. The SMTP transactions may consist of a variety of commands and responses, in a variety of sequences, and the format is defined by RFC822 [22]. 


\subsection{SMTP Characteristics}

The traffic generated by SMTP is a combination of protocol messages and the contents themselves. The sender and recipient MTAs behave as ON-OFF sources. The ON periods are determined by protocol message elements and user message contents. The OFF periods depend on the response from the peer process, thus giving rise to a lock-step behavior. In order to characterize such traffic it is therefore necessary to look at the content sizes generated at different organizations and identify the common features.

The process of modeling the SMTP traffic is essentially aimed at finding specific properties across organizations, regions, etc. The message contents themselves may have local characteristics but the content sizes may still be statistically similar.

In order to develop a simulation model for SMTP, a set of key properties have been identified. Using the software tools described previously, the statistics about the key elements have been collected, as follows:

- Session arrival timings. These statistics indicate the intensity of SMTP traffic originating from an initiator host directed towards a responder host.

- The number of e-mail transactions within a session. This characteristic indicates the e-mail traffic intensity between a specific host pair. Multiple message transactions within a session between two hosts may occur due to the frequent genuine interactions between the user groups at the two sites. It may also occur if for some reason the hosts in question can not connect for a while and thus there is a resultant message queue that builds up at the initiator end.

- Message body lengths. These statistics describe the payload traffic. The traffic can be simple textual messages typed in by the users or even files, audio, video material, word processor documents, etc. All these are specifically marked as attachments and specially encoded to be packed into a single message body. To characterize the distributional properties of the message body sizes, it is imperative to look into application layer payload information. Because of the personal and sensitive nature of this operation, it is not always possible to adopt this methodology to collect information from different organizations. However, there is an alternative method that involves the analysis of the SMTP responder logs. Without having access to the message contents themselves, these logs can be used instead to collect the message body sizes and the number of recipients each message is addressed to.

- Message timings. These are the timestamps at which application layer messages are observed by the monitor. These messages can be protocol exchanges or the message content themselves. By collecting the timings for the client commands and the server responses, the ON and OFF durations nature of the SMTP sources can be determined.

\subsection{Traffic Analysis}

Generally, the SMTP exchanges have been observed to exhibit a bimodal structure [19, 25], and these results are confirmed by our study. The control messages are short messages (often less than 50 bytes) whereas the message bodies are bigger and may even give rise to multiple packets at the link layer. Some of the main results of this analysis are as follows:

- The arrival process of user sessions for wide-area traffic has been previously shown to be well modeled by Poisson processes [20]. Evaluating the monitor timings for SMTP connection initiation inter-arrival times in the observed data sets, we have modeled this process as an Exponentially distributed random variable with a mean of 20 seconds.

- For simulation purposes, we have also considered the analysis of application protocol messages between the initiator and the responder. This study has revealed that they have a very simple structure (table 1), e.g., the first seven message lengths can be adequately modeled by a Uniformly distributed random numbers with different ranges. It is also observed that protocol commands like "MAIL FROM" and "RCPT TO" have Uniform distributions with different values, and this indicates that they are highly dependent on characteristics such as the lengths of usernames and hostnames. Furthermore, other several messages are observed to have constant sizes, due to the typical protocol structure of SMTP.

- The process of fitting a distribution model to the message body was initiated with the investigation of the complementary distribution plot for the message body sizes, as shown in figure 1. We observe the mixture of two different distributional components, one for the bulk of the distribution and another for the upper tail (power-law characteristics). 


\begin{tabular}{|c|c|c|}
\hline Msg type & $\begin{array}{c}\text { min, median, mean, max } \\
\text { (in bytes) }\end{array}$ & Modeled as (range) \\
\hline \hline INIT resp & $25,87,87.13,96$ & Uniform (20-100) \\
\hline HELO cmd & $19,23,23.18,32$ & Uniform (20-50) \\
\hline HELO resp & $25,175,160,187$ & Uniform (120-300) \\
\hline MAIL FROM cmd & $30,45,42,68$ & Uniform (25-75) \\
\hline MAIL FROM resp & $30,43,41.48,86$ & Uniform (30-80) \\
\hline RCPT TO cmd & $15,35,34.97,59$ & Uniform (15-60) \\
\hline RCPT TO resp & $10,47,46.37,74$ & Uniform (10-75) \\
\hline DATA cmd & 6 & Constant \\
\hline DATA resp & 45 & Constant \\
\hline DATA_END cmd & 3 & Constant \\
\hline DATA_END resp & 45 & Constant \\
\hline QUIT cmd & 6 & Constant \\
\hline QUIT resp & $27,37,38.71,45$ & Uniform (25-50) \\
\hline
\end{tabular}

Table 1: Characteristics of SMTP protocol message sizes

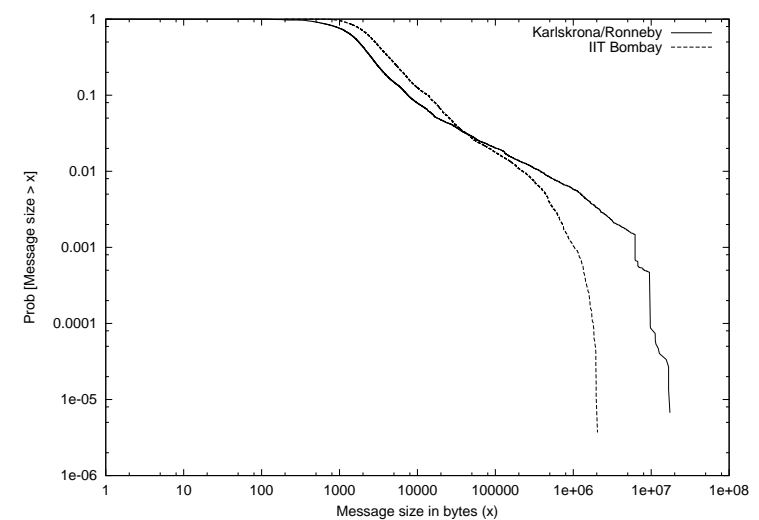

Figure 1: CCDF of SMTP message body sizes (log-log scale)

- The next step is to verify the hypothesis about the participating distributions and determine the cutoff point between the two participating distributions. A graphical technique was used as a first step. The procedure is illustrated for the message bodies from IIT Bombay only. A CDF plot for the message body sizes up to 10000 was investigated (right plot of figure 2), using a linear scale. We observe a linear behavior for message sizes between 500 and 3000, indicating a Uniform behavior (left plot of figure 2).
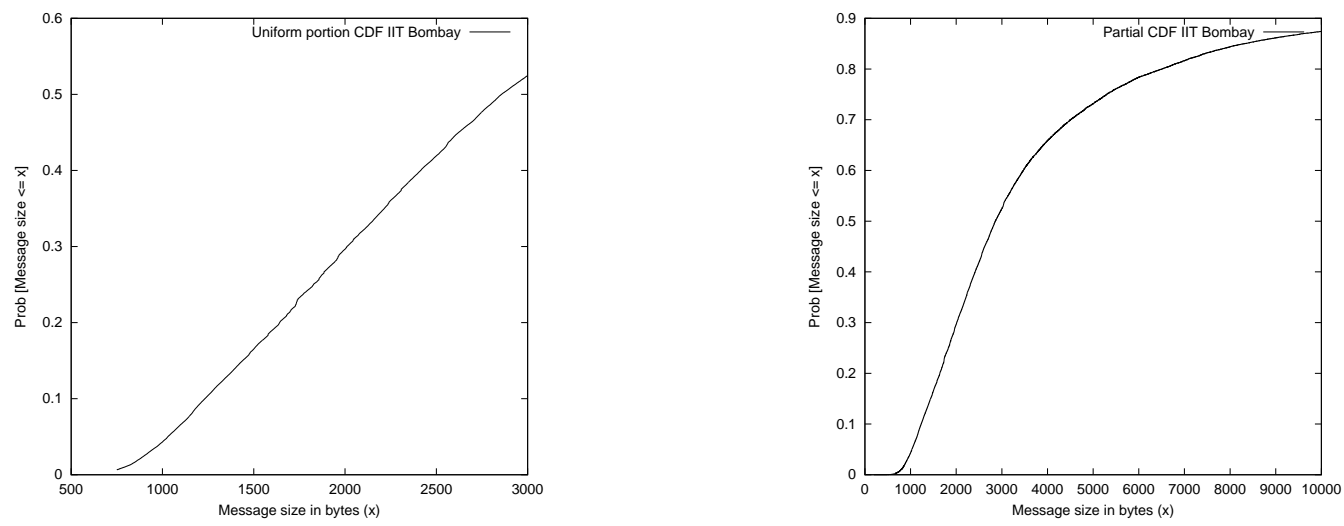

Figure 2: SMTP message body sizes from IIT Bombay: up to 3000 bytes (left) and up to 10000 bytes (right)

- Using the information obtained from the graphical procedure, successive censoring technique was applied. The body and tail portions were tested for Uniform and Pareto distributions respectively. The AndersonDarling (AD) test was applied repetitively on the appropriate subsamples. In a run of 100 tests, it was possible to detect a significance level of 0.05 at least in $50 \%$ of the cases and $75 \%$ of the cases showed a significance of 0.01 . Subsample sizes up to 150 showed this behavior. Higher subsamples showed only 
traces of significance. This behavior indicates that the models are not statistically exact. As a result, it can only be concluded that the models are at least approximately correct.

- Using the previous step, the cutoff point was determined. Having obtained the cutoff point, the maximum likelihood parameters and the $\lambda^{2}$ discrepancy value were obtained. In cases where multiple points for significance tests showed similar values, the model with minimum discrepancy was chosen. Electronic message exchanges are rather personal and to that extent they may represent some kind of socio-cultural variations. However, the messages sizes of the collected data sets have been observed to show statistically invariant properties. The actual message body sizes and the corresponding fitted model for the dataset collected from IIT Bombay is shown in figure 3. The dataset from BIT, Karlskrona also shows similar structure. The solid line shows the complementary cumulative distribution function for the real data and the dotted line shows the model fitted to the data. The results of the fitting process are shown in table 2 together with the parameters of the Uniform distribution ( $a$ and $b$, the lower and upper limits of the Uniform random variable) and Pareto distribution (the shape parameter $\alpha$ and the location parameter $\beta$ ). Finally, we also observe the disturbing phenomenon of $\alpha<1$, indicating unstable processes in SMTP message sizes. Such values have been disregarded in the simulation model.

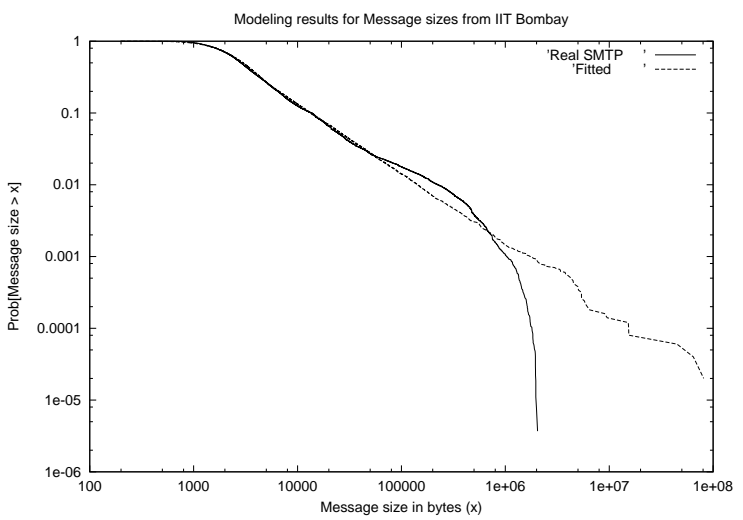

Figure 3: CCDF for the message body sizes vs fitted model (log-log plot).

\begin{tabular}{|c|c|c|c|c|c|c|}
\hline Orgn & $\begin{array}{c}\text { 1st Qn, median, mean, 3rd Qn } \\
\text { in bytes }\end{array}$ & $\begin{array}{c}\text { Subs } \\
\text { size }\end{array}$ & $\begin{array}{c}\lambda^{2} \\
\text { value }\end{array}$ & $\begin{array}{c}\text { Uniform } \\
\text { parameters }\end{array}$ & $\begin{array}{c}\text { Pareto } \\
\text { parameters }\end{array}$ & $\begin{array}{c}\text { Body } \\
\text { mass }\end{array}$ \\
\hline \hline Bombay & $1828,2857,12090,5292$ & 100 & 0.11 & $\begin{array}{c}a=500 \\
b=3000\end{array}$ & $\begin{array}{c}\alpha=0.99 \\
\beta=3000\end{array}$ & $m=0.52$ \\
\hline Karlskrona & $1028,1766,11660,2015$ & 100 & 0.09 & $\begin{array}{c}a=100 \\
b=2150\end{array}$ & $\begin{array}{c}\alpha=0.79 \\
\beta=2150\end{array}$ & $m=0.606$ \\
\hline
\end{tabular}

Table 2: Model parameters for the message sizes.

- E-mail messages are primarily used for inter-personal communication. However, with the advent of MIME encodings, e-mail is increasingly being used to send files as attachments to the mail body. The bimodal behavior appears to be caused by this mixture. The simple body messages without any attachments are likely to be the cause of the Uniformly distributed body portion. The attachments to the main body are likely to generate the Pareto tail portion. Further investigation of the message body contents is rather difficult. First, to be able to segregate the various body attachments, these have to be decoded using MIME. Second, it is rather controversial to look into the contents because of the personal and confidential nature of the messages bodies.

- In the collected SMTP packet traces multi-trasaction SMTP sessions were not observed. However, it is mentioned that they can occur when there is large amount of SMTP traffic between a designated host pair.

\section{HTTP}

The WWW application is accessed typically through Web browsers. The human user initiates the transfer of information from a server by clicking on links in a Web document. A Web page consists of diverse objects. The (content) objects are transferred from the WWW server to the client. On the server, each of the objects 
is physically stored as a distinct file such as HTML file, GIF or JPEG image, audio clip, etc. The collection of all servers on the global Internet creates an information Web. On this Web, each object is associated with a unique address. The logical addressing scheme of the Web objects is defined using a mechanism called Uniform Resource Identifier (URI) [4].

The HTTP application layer protocol $[4,9]$ is at the core of the WWW information network. The protocol uses TCP as a transport mechanism. Characterization of the WWW services essentially involves characterization of the objects transferred using HTTP. The Web means different things to different people. One server may be a newspaper type, a second one may be an university type, and the third one may be a site geared to provide entertainment. The content types may be different in each case. Thus, in order to characterize the Web objects, it is important to analyze the objects resident at a wide cross section of Web servers. Furthermore, another important issue is the user preference. The network traffic and host processing load at a server is mainly determined by the number of concurrent users. This is a highly subjective issue and thus difficult to characterize.

\subsection{Session Structure}

The HTTP session starts when the client sends a request to the server for a specific document. The server processes the client requests and uses the TCP connection opened by the client to send back the results. It is not aware of the structuring of the Web pages. It is only the browser on the client side that interprets the fetched pages to find out the structure and to determine the representation on the browser screen.

A typical format used for the HTTP GET request and the corresponding response is shown in fig. 4. The header fields have important roles to play during HTTP protocol exchanges. The headers in the HTTP requests have fields to indicate the capabilities of the browser so that the server can choose the appropriate version of the object. The header also contains information about the current version object (if it has been fetched in the past) specified via the If-modified-since field. Finally, the request header field Connection contains information about the HTTP protocol version and its specific options. The version and the option settings are used by the server for keeping the transport connection open or close it after each object. The headers in the HTTP responses identify the content type, contain information about its length, and the encoding methods so that the browser can apply the appropriate interpretation rules. The response header also contains the response code. One important use of the response code is to signal to the browser to fetch the object from an alternative URI. The response header may also contain the Last-Modified field so that the browser may update its in-cache copy. The Connection field in the response header indicates the persistent HTTP capabilities of the server. The persistence capabilities of the client and the server have to be compatible. The advanced features of HTTP become activated only under these circumstances. Otherwise, the transfers fall back to the default non-persistent HTTP mode.

Most Web pages are designed around an object called main (primary) object. The main object serves as an anchor and holds, in its body, several references to other URIs. These references may be links or even embedded (secondary) objects such as inlined images. The links are underlined or marked with special colors or symbols when they are rendered on the user screens. These are hints to the human user so that the user can further click on them and fetch them. On the other hand, the participating URIs corresponding to the embedded objects are implicitly fetched and rendered on the user screen. Typically the embedded objects are of type images, maps, frame layouts, audio clips, etc. Very often, the embedded references are located on the same server as the main page but in a few cases they reside on a different server and have to be fetched from there. The body of a typical main object is shown in figure 5. The HTTP body immediately follows the response header in the HTTP reply with a blank line separating the header and the body.

The body of the server response contains text mixed with tagged objects to identify HTTP items. Some of the HTTP items are hyperlinks. Typically these are the items that are rendered underlined or highlighted items so that the user can further click on to download. Such items are not embedded objects. They require explicit user action to initiate their transfers from the respective servers. In addition, there are HTTP elements that do not require any user action and are implicitly fetched by the browser automatically without any explicit action on the user part. Fig. 5 illustrates some of the typical tagged items together with their respective categories extracted from actual Web pages. Notice the further classification of the embedded objects as "Internal" and "External". There are other types such as CODE, BODY, BGSOUND etc, which also represent embedded objects.

A stochastic marked point process is used to model the HTTP session and the associated timings (fig. 6). A HTTP session is defined as being the transaction for downloading a single Web page, with associated main and secondary objects. Aborts are not considered in our model. It is also mentioned that the spidering of Web sites is used, in our model, to analyze the static content structure and sizes that reside at diverse Web servers. As a result, we do not address the dynamics of Web access processes. However, dynamic Web flows are implicitly created in our simulation model when the user selects a subset of the static content from a Web server.

The following variables are considered for a session: 


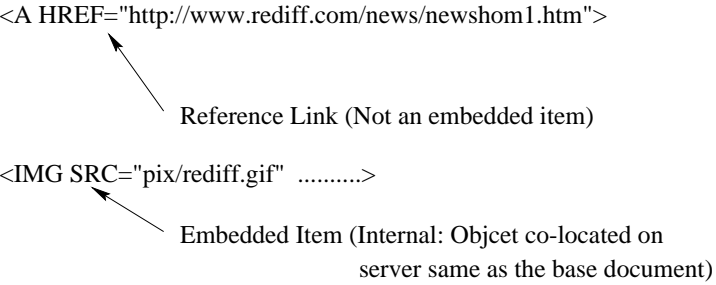

Figure 4: Contents of a typical GET request header (top) and the corresponding response (bottom)

- ON time: time duration elapsed for the fetching of the main object and the associated embedded objects.

- active OFF time: time duration between consecutive fetches of embedded objects (within same ON period), and

- passive OFF time: time duration elapsed between the completion of the transfer of one page and the beginning of the following session (user think time).

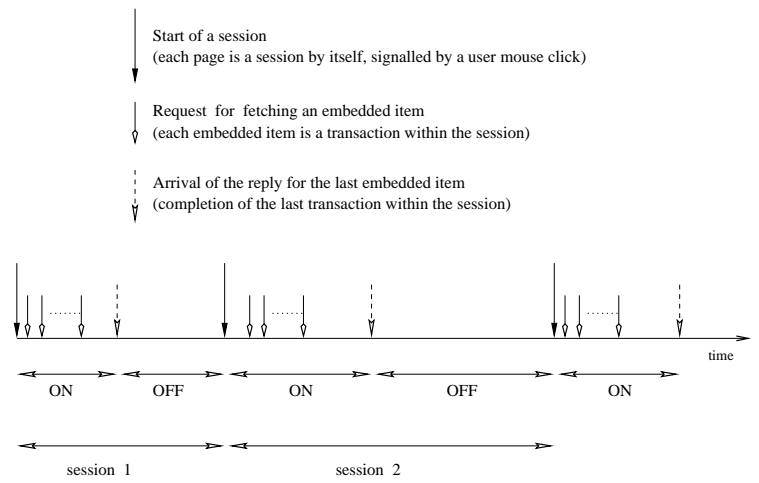

Figure 6: HTTP session

For the purpose of explaining the data collection and modeling aspects, a session is defined to start at the instant the user asks for a URI by explicitly naming it or by pointing the mouse at a link reference and clicking on it. With this epoch, the client side connects to the server and starts sending the request for the main object and subsequently the embedded objects. The server processes the requests and sends the contents back to the client. The session is said to have ended when the last embedded object is received at the client side. At this point, the view on the browser screen is complete and the human user starts reading the page. From the traffic point of view this is a silent period, which we define as the inter-session gap (i.e., passive OFF time). This gap represents the human user behavior (user think time). It is the actual time duration spent by the user with the page and typical action may simply involve browsing, printing, or even saving it on local disk for future reference.

\subsection{HTTP Characteristics}

Using the software tools described in section 2, the statistics about the key elements have been collected. Some of the most important elements are as follows:

- Session arrivals. These statistics indicate the intensity of WWW requests generated by users. 
- Number of transactions within a HTTP session. This is essentially given by the number of embedded objects inside the Web page that constitutes the session. This further determines the session durations. The larger the number of transactions, the larger the number of transfers that have to be performed to complete the session.

- Request message lengths. The request messages from the client to the server are usually short messages that are transmitted for fetching each of the Web objects whether they are of type main or of type embed$d e d$. These messages and their timings determine the traffic stream that is generated from the client to the server.

- Reply message headers. The WWW reply messages from the server to the client consist of a header portion and the content portion. The statistics of the reply header sizes is an important item that needs to be characterized for evaluating the traffic created in addition to the content portion. Furthermore, the reply headers themselves have important information that needs to be collected and characterized.

Some of the most important attributes that can be generated by studying the reply headers and the content are as follows:

- Statistics about the success or failure of WWW access requests, which can be generated by using the reply code information in the reply message header.

- The percentage of the requested items that were not transferred due to caching on the client side. This can be obtained by studying the Last-Modified field in the reply header.

- The type of objects transferred. When the client side sends a request, the only information it has is the URI. The classification of the objects into HTML or non-HTML can be done by studying the Content-type field in the reply header from the server.

- The content lengths. This is the main contributor to the traffic from the server to the client.

\subsection{Traffic Analysis}

WWW is a server-centric application that generates most of the data flow from the server towards the clients. The server therefore has some kind of latent properties in terms of content pages it holds as well as the structural properties of these pages. The structural properties refer to the number of embedded objects in a page, the types of embedded objects, etc. Some of the latent properties of a server are exhibited during WWW sessions when the user first selects a specific server and then from that particular server it chooses a portion out of the total domain on WWW pages. In terms of analogy, the situation is similar to the energy systems where the objects, by virtue of their location, have potential energy. Some of the potential energy gets converted into kinetic energy when the object moves. Accordingly, one of the main goals of this experiment was to study both the latent and the exhibited properties in the WWW application. The latent properties are captured using the GetWeb software whereas the exhibited properties are collected using the http flowstat program. The main characteristics of the WWW application are as follows:

- The statistics of WWW session arrivals, which are determined by the user behavior. A WWW session starts when a user requests a main object and may contain several embedded object requests as subsequent transactions as well. However, data collected from the real Internet access links shows the aggregate behavior of hundreds of clients and the analysis of one day's collected traces using http flowstat shows the generation of thousands of connections. The difficulty therefore does exist of how to distinguish between requests for main object and for embedded objects, by only analyzing the flows captured from the network. However, we have observed that, in a few cases, the silence periods between successive WWW requests are large enough to make this distinction. We have therefore assumed (for simulation purposes) that any inter-arrival gap larger than 5 seconds is an inter-session gap. As per observations so obtained, the arrivals of requests for WWW sessions (at the user level) can be reasonably well modelled by a renewal process, and in most of cases they are simply a Poisson process [11]. The inter-arrival time between requests for main objects has been observed to be well modeled by an Exponential process with a mean of 15 seconds.

- The inter-arrival time gaps between successive WWW requests belonging to a given WWW session, which represent the active OFF times of the WWW client (fig. 6). This parameter is obtained from the output of the http flowstat software and it has been clearly observed that these timings are in the range 100-500 milliseconds. These timings are mainly dependent on the HTTP version and the pipelining feature of the 
browser software. With pipelining, the client software transmits all the requests one after another without waiting for the results of the previous request to arrive. In such cases, the active OFF times tend to be very small. This is the case with HTTP/1.1 and HTTP/1.0 with persistence mode operation. Even in case of HTTP/1.0 with no persistence, the client may open several TCP connections in parallel and send out one HTTP request on each of these connections.

- The request message sizes were collected from the output of the http flowstat program as well. Compared to the bimodal distribution observed in [16], the analysis of the message sizes in our traces reveals instead that this parameter can be well modeled as a Uniformly distributed random variable in the range 180-600 [11]. Figure 4 shows the structure of the request headers. For a browser like NETSCAPE, the limited variability seen in the sizes of request messages is mainly contributed by the first line showing the URL, the second line containing the caching information, and the fifth line containing the domain name of the server. Similarly, the header sizes of the response messages also show limited variability. This parameter can be modeled by a Uniform random variable in the range 250-700.

- The models for HTTP object sizes (collected through Web mirroring software) are presented in table 3. The CCDF plots for the server categories are shown in figures 7 to 9. The Web pages have been fitted as a mixture of Lognormal (with parameters $\mu$ and $\sigma$ ) and Pareto distributions (with parameters $\alpha$ and $\beta$ ). The quality of fitting for the two candidate distributions are shown in the 'Remarks' column of table 3 . The remarks $(L o k)$ and (L poor) refer to the Lognormal body portion. Similarly, the remarks (Pok) and (P poor) refer to the Pareto tail. These remarks are based on the significance levels passed by the censored AD test. The choice of subsample sizes for applying the AD test in each case is also shown. The collected observations have been grouped into HTML and non-HTML types and their relative frequencies are also shown. It is observed that this model does not fit well in some cases. In a majority of cases however, it appears that the tail portion comes from a Pareto process. However, in some cases (two of the news/media servers), the power-law behavior is negligible. In such cases, the complete distribution can be well represented as a Lognormal random variable only. Such cases are highlighted with a '*' in the 'Remarks' column. Furthermore, in the case of commercial organizations, the Lognormal distribution seems to be a poor choice to fit the body portion. This is a disturbing result and as such it will be further investigated. The HTML and non-HTML groups, when plotted separately on the CCDF plot, reveal that this behavior appears to come from the HTML type documents mostly (figure 10). From the CCDF plot it also appears that in such cases the body portion can be approximated by a Uniform distribution. This feature seems to be common to all the commercial servers investigated.

- The analysis of the HTTP object sizes collected on the client side via NetVCR ${ }^{T M}$ monitor is shown in table 4. This is a typical case seen in the access networks (staff and students) at Blekinge Institute of Technology. A representative set of results are shown in the table. The CCDF plots for the sizes of Web pages accessed in the two subnets is shown in figure 11. A notable feature of the results shown here is that the qualitative aspects of the model fitting are much better than shown in table 3 and hence they are not been reported.

- A deeper analysis of the traffic seen on the staff access network is shown in figure 12. The density of HTTP traffic on this network is rather low. In addition, the access pattern is rather repeatative. In a broad sense, every day the traffic is drawn from a few servers and almost the same set of pages. A further investigation reveals that the major contribution to the traffic comes from a basic set of Web servers that includes newspapers, technology sites, etc. The caching percentage has been observed in this case to be about $10 \%$. The success rate of Webpage access is about 95\%. When the Web pages are grouped and studied separately (figure 12), then it is seen that the HTML type pages accessed on the staff network have negligible power law behavior. The non-HTML pages show some power law behavior but the intensity is quite low. This may be attributed to the low variability in the Web pages accessed on the staff network.

- The density of HTTP traffic on the student access network is expectedly higher (figure 13). On a typical day, the student net may show about $350 \mathrm{MB}$ of HTTP traffic. In addition, the analysis of the access pattern shows a richness in variety both in number of Web sites and content types (such as audio, graphics, java). The caching percentage has been observed to be lower in comparison to the staff access net and it is about $4 \%$. The success rate of Webpage access is about $90 \%$. When the Web pages are grouped and studied separately (figure 13), the higher intensity of power law behavior is apparent in both the HTML and non-HTML categories. 


\begin{tabular}{|c|c|c|c|c|c|c|c|c|c|c|c|}
\hline Site Name & $\mu$ & $\sigma$ & $\alpha$ & $\bar{\beta}$ & $\begin{array}{l}\text { Tail } \\
\text { mass }\end{array}$ & $\begin{array}{l}\text { Signif } \\
\text { sub } \\
\text { size }\end{array}$ & $\begin{array}{l}\lambda^{2} \\
\text { value }\end{array}$ & $\begin{array}{l}\text { cutoff } \\
\text { point } \\
\text { (byte) }\end{array}$ & $\begin{array}{c}\text { HTML } \\
\%\end{array}$ & $\begin{array}{c}\text { non } \\
\text { HTML } \\
\%\end{array}$ & Remarks \\
\hline www.cs.berkeley.edu & 8.49 & 1.86 & $\overline{0.766}$ & 5717 & 0.13 & 200 & 0.06 & 81435 & 44 & 56 & $($ Lok)(Pok) \\
\hline www.hk-r.se & 7.97 & 1.27 & 0.624 & 1160 & 0.24 & 200 & 0.09 & 11394 & 58 & 42 & $($ Lok)(P Po $)$ \\
\hline www.cs.purdue.edu & 7.09 & 1.27 & 0.83 & 1547 & 0.15 & 200 & 0.06 & 15137 & 74 & 26 & $(\mathrm{Lpo})(\mathrm{Pok})$ \\
\hline www.cam.ac.uk & 8.12 & 1.28 & 1.03 & 1860 & 0.16 & 200 & 0.02 & 10964 & 68 & 32 & $($ Lok $)($ Pok $)$ \\
\hline www.uiuc.edu & 8.01 & 1.30 & 1.62 & 3865 & 0.17 & 200 & 0.04 & 11526 & 69 & 31 & $($ Lok $)($ Pok $)$ \\
\hline www.hp.com & - & - & 0.46 & 54 & 0.04 & 100 & 0.12 & 53658 & 40 & 60 & $($ Lpo $)($ Pok $)$ \\
\hline WWW.ibm.com & - & - & 0.63 & 418 & 0.06 & 100 & 0.13 & 36096 & 61 & 39 & $($ Lpo $)($ Pok $)$ \\
\hline www.nokia.com & - & - & 0.51 & 142 & 0.06 & 100 & 0.13 & 35428 & 34 & 66 & $(L p o)(P o k)$ \\
\hline www.lucent.com & - & - & 1.12 & 16578 & 0.06 & 100 & 0.14 & 199961 & 60 & 40 & $($ Lpo $)(P o k)$ \\
\hline www.rediff.com & 8.50 & 1.56 & - & - & - & 100 & 0.05 & - & 67 & 33 & $*$ \\
\hline www.nytimes.com & 8.85 & 1.88 & 1.85 & 8423 & 0.29 & 100 & 0.17 & 16408 & 72 & 28 & $($ Lpo $)(P o k)$ \\
\hline www.the-week.com & 9.78 & 0.64 & - & - & - & 100 & 0.05 & - & 34 & 66 & $*$ \\
\hline www.india-today.com & 8.90 & 1.53 & 1.46 & 6160 & 0.13 & 300 & 0.05 & 24824 & 50 & 50 & $($ Lok)(Pok) \\
\hline
\end{tabular}

Table 3: Modeling results for HTTP object sizes collected from different categories of Web servers

\begin{tabular}{|l|l|c|c|c|c|c|c|c|c|c|}
\hline Karlskrona & Type & $\mu$ & $\sigma$ & $\alpha$ & $\beta$ & $\begin{array}{l}\text { Tail } \\
\text { mass }\end{array}$ & $\begin{array}{l}\text { Signif } \\
\text { sub size }\end{array}$ & $\begin{array}{l}\lambda^{2} \\
\text { value }\end{array}$ & $\begin{array}{l}\text { cutoff } \\
\text { point }\end{array}$ & $\%$ \\
\hline \hline \multirow{2}{*}{ Staff Net } & HTML & 8.54 & 1.60 & - & - & - & 300 & 0.06 & - & 45 \\
\cline { 2 - 11 } & non HTML & 7.71 & 1.68 & 1.69 & 3178 & 0.14 & 300 & 0.04 & 10112 & 55 \\
\hline \multirow{2}{*}{ Student Net } & HTML & 6.64 & 1.50 & 1.30 & 1418 & 0.14 & 200 & 0.04 & 11394 & 30 \\
\cline { 2 - 11 } & non HTML & 7.08 & 1.87 & 0.93 & 1147 & 0.22 & 200 & 0.06 & 5802 & 70 \\
\hline
\end{tabular}

Table 4: Modeling results for the HTTP object sizes collected through packet traces

\subsection{Structure of Web Pages}

The structure of Web pages is characterized in terms of statistical properties of the number of embedded objects as well as their respective types (HTTP vs non-HTTP). In other studies focused on generation of sythetic workloads $[2,3]$, the structure of Web pages is inferred from application session logs. This strategy is adequate in an environment where the application logs represent the access activity of one user. However, this may not reflect the situation when the access logs pertain to the activities of a large group of users. We therefore use another method, i.e., the so-called Direct Probe method [11], to infer about the structure of the Web pages.

Further, the structure of the content pages is likely to be a property of the server itself. This feature represents the qualitative design aspects of the content itself and to that extent reflects the properties of the specific organization. Intuitively, it is expected that the Web page design features at the universities may be different from those designed for commercial purposes. It is expected that the differences are both quantitative and qualitative. For instance, the density of embedded objects is expected to be higher in the case of news/entertainment and commercial sites. On the other hand, the bulk of pages on newspaper sites will be of HTML type and in terms of sizes they should be small. As far as the university sites are concerned, these are expected to be rich with research reports having Postscript or PDF format. These files tend to be large in size and therefore contribute towards the power-law behavior in the tail. While these intuitive arguments are difficult to prove rigorously (because of the subjective human factors involved), they are, to a limited scale, demonstrated in figures 7 to 9 .

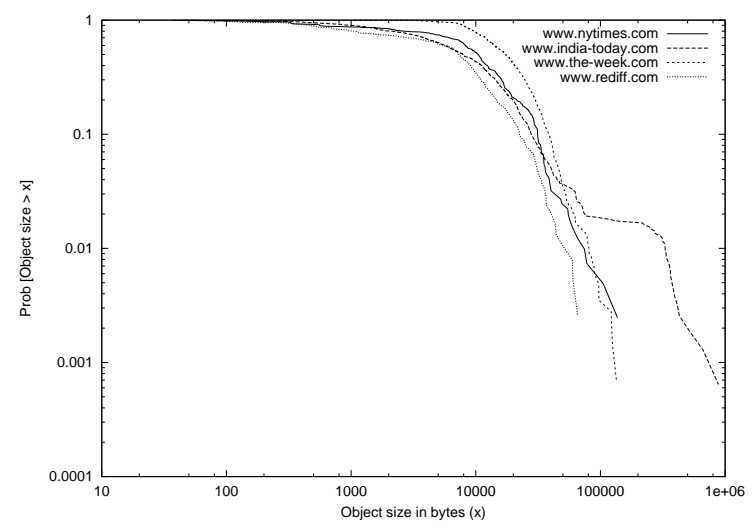

Figure 7: CCDF of entertainment servers 


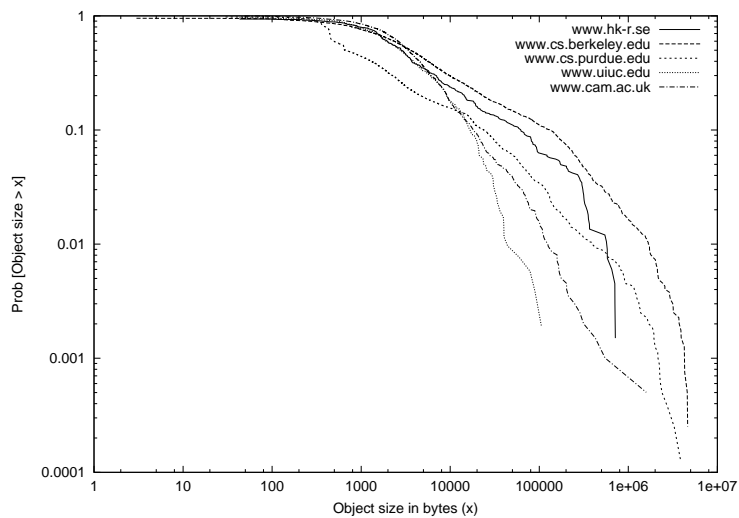

Figure 8: CCDF of educational servers

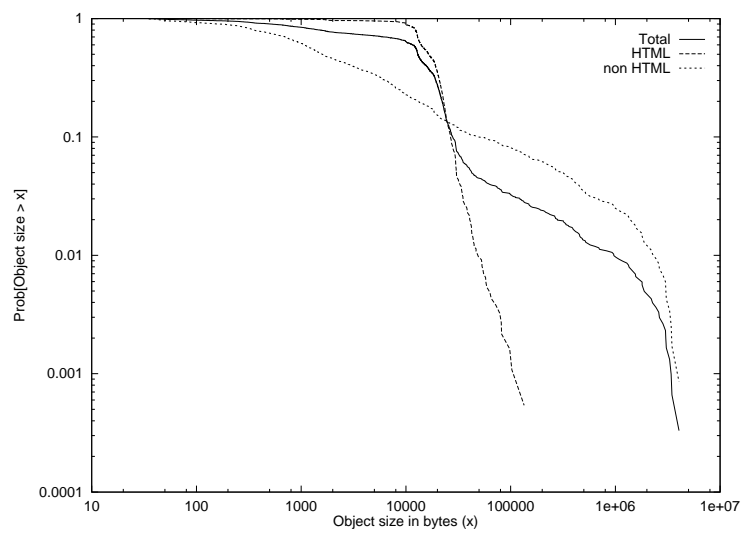

Figure 10: CCDF of ibm.com servers

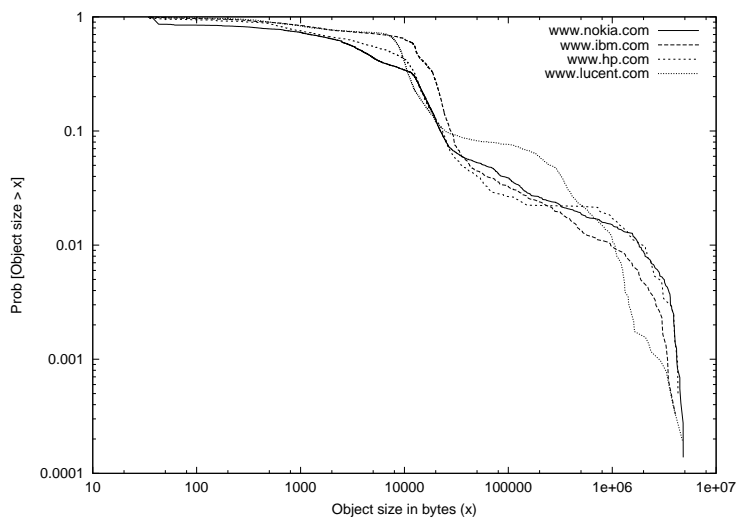

Figure 9: CCDF of commercial servers

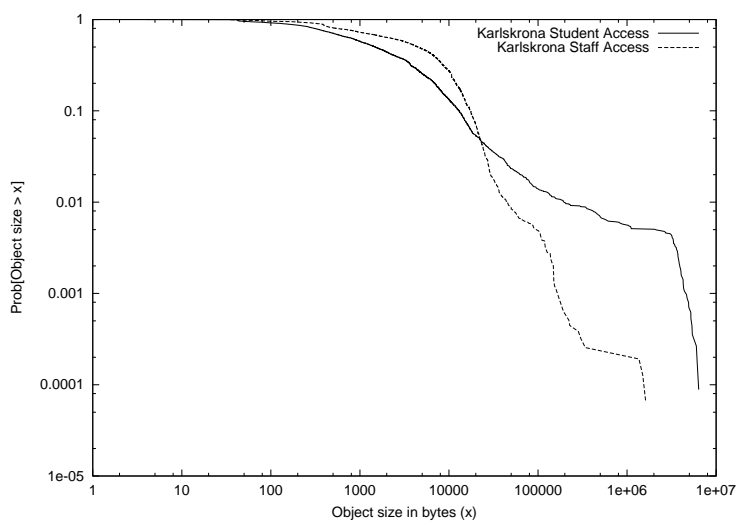

Figure 11: CCDF of Karlskrona

Based on this, and on our collected data traces, we have developed a stochastic model for the number of embedded objects that may occur in a Web page. The following steps describe the modeling methodology:

- The basic dataset used in the modeling process is the output of the GetWeb software that probed a large number of WWW servers and recursively downloaded their content pages.

- The data generated in the previous step was grouped using two levels of indexing, the serverid and the pageid. The design of indexing mechanism was geared towards the goal of retaining the server information and also the particular page information within the specific server. It was observed that in many cases a single embedded object occured many times (e.g., the GIF files representing the bullets in a HTML page layout). The modeling process counts the contribution of such multiple occurances as 1 . In other words, the modeling procedure only considers unique embedded objects and rejects duplicate occurances of these objects. This corresponds to a realistic scenario because after the GIF object is downloaded the first time, it is available in the local cache, and hence it is not downloaded again.

- Subsequently, the literature in applied statistics was searched to find the statistical modeling of a real-life situation that closely resembles the problem at hand.

Based on these facts, a mathematical model for this very specific case has been built [15], which is useful for simulation purposes. The number of embedded objects has been found to be well modeled by a Negative Binomial distribution (Fig. 14). This distribution is used, for instance, to find the number of Bernoulli trials needed to find the r-th success. Furthermore, it has been also shown that the Negative Binomial distribution can be well approximated as a mixture of Poisson distributions where the expected values of the Poisson distributions vary according to a Gamma distribution [13].

Accordingly, a simple model of the number of embedded documents in a Web page can be as follows. Assume that, on a specific server $i$, the number of embedded objects in the Web page $j$ is $X_{i, j}$. This number is conditioned by the intensity $\lambda_{i}$, which is a server characteristic that represents the density of presence of embedded objects in the Web pages resident at server $i$. In other words, the quantity $\lambda_{i}$ is implicitly fixed when 


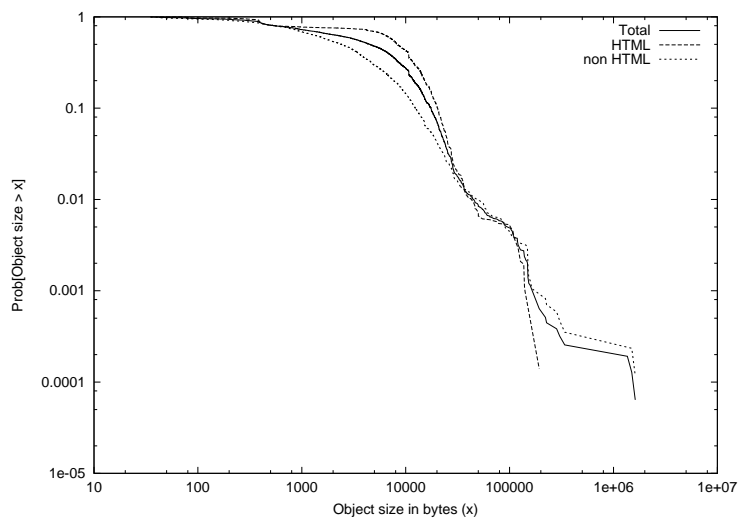

Figure 12: CCDF of Karlskrona, staff only

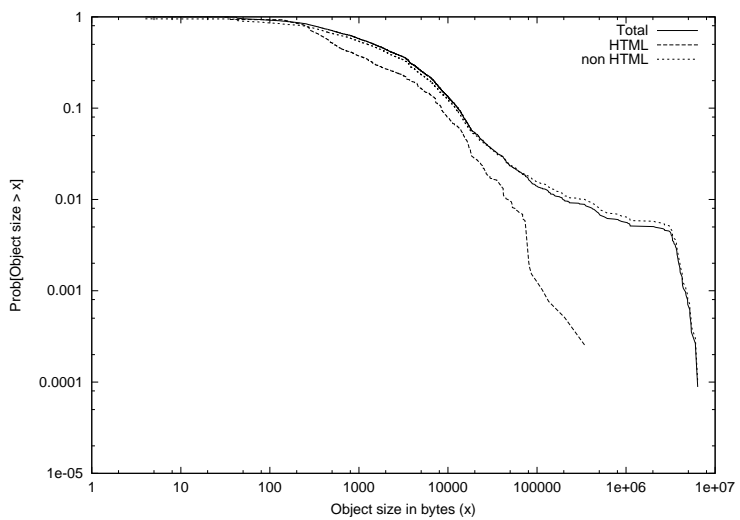

Figure 13: CCDF of Karlskrona, students only

the user selects a particular server during a Web session. The (conditional) distribution $F(X \mid \lambda)$ of the number $X$ of embedded objects in a Web page can be modeled as:

$$
F(X \mid \lambda) \sim P(\lambda)
$$

where $P$ represents the Poisson process. From the collected observations (regarding the total number of embedded objects occuring in a page), the number of pages having zero embedded objects is excluded. Consider $C$ as being the fraction of these pages. The remaining observations (having the number of embedded objects greater than zero) can be further used to generate a distributional model by using Gamma distribution with shape parameters $\alpha$ and $\beta$. In other words, the random process $X$ occurs as a mixture of Poisson distributions and the expected intensity $\lambda$ of every Poisson distribution vary according to a Gamma distribution with PDF:

$$
f(\lambda)=\frac{\lambda^{(\alpha-1)} \exp \left(-\frac{\lambda}{\beta}\right)}{\beta^{\alpha} * \Gamma(\alpha)}
$$

where $\Gamma(\alpha)$ is the Gamma function (also known as Euler function) of variable $\alpha$ and the parameters $\lambda, \alpha$ and $\beta$ are larger than zero. Under these conditions $X$ has a Negative Binomial distribution (with parameters $\alpha$ and $\beta$ ) and the PDF of the number of embedded objects is [13]:

$$
\begin{gathered}
\operatorname{Prob}[X=x]=\int_{0}^{\infty} f(\lambda) \frac{\lambda^{x} \exp (-\lambda)}{x !} d \lambda= \\
\left(\begin{array}{c}
\alpha+x-1 \\
\alpha-1
\end{array}\right)\left(\frac{\beta}{\beta+1}\right)^{x}\left(\frac{1}{\beta+1}\right)^{\alpha}
\end{gathered}
$$

A model for the generation of $X$ is as follows:

- Generate a Uniformly distributed random variable $\omega$ in the range $0-1$. The value $C$ can be estimated from the observations (direct probe) and in our case it has been estimated to be 0.1202 . If $\omega \leq C$, then return the number of embedded objects as zero. Otherwise continue with the next step.

- Generate a random number $N$ from the Negative Binomial distribution. From the collected data it was observed that the maximum number of embedded objects in a page is 75 . Also, there were about $6 \%$ of the cases where the embedded objects were located outside the server hosting the main object. Using these observations, the value of the parameters of the distribution obtained via the maximum likelihood estimation are $\alpha=1.386$ and $\beta=6.985$.

Fig. 14 shows the observed CDF of the number of embedded objects (as collected from our real Web pages) against the distribution function obtained by the modeling process presented above.

\section{$7 \quad$ FTP}

The transfer of files between hosts has been one of the most popular Internet services. In its original form, the FTP service [23] was controlled using the userid-password mechanism and thus had a personalized orientation. 


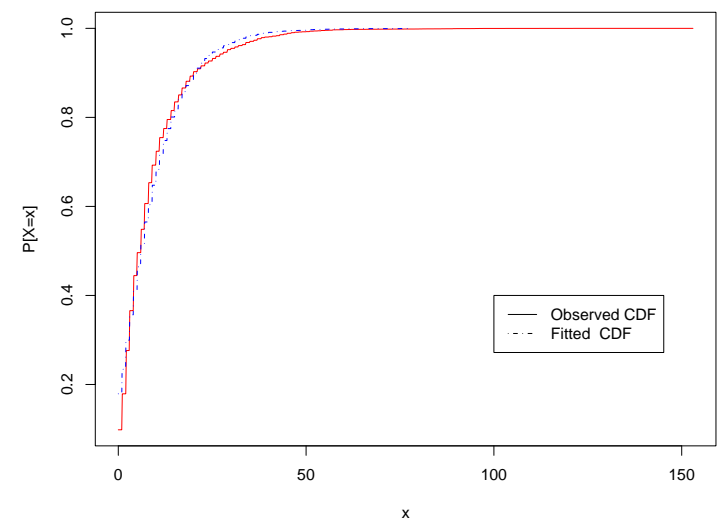

Figure 14: CDF of the number of embedded items

In order to have access to a remote filesystem, the user needed to have a userid on the remote machine. However, the anonymous FTP service has alleviated the need to have a userid-password on the remote machine. In fact, this service was very popular for sharing information, data, program code, etc., among the scientific community until the arrival of HTTP. Over the recent years, HTTP has provided an alternative mechanism for distributing information content and slowly it has been taking over the role traditionally played by FTP. FTP however still continues to be one of the basic Internet applications in use today.

Both FTP and HTTP enable file transfer between hosts. However, there are differences between them, e.g., regarding the out-of-band control channel. In the case of HTTP, the control information travels on the same connection as the data. On the other hand, FTP uses two parallel channels. The first is the control channel that is a TCP connection established between an ephemeral port on the client host to port 21 on the server. This channel is used for authentication of user credentials and transmission of commands, which are organized in the form of specific protocol messages between client and server that control the file transfer operations.

The actual transfer of files (or even the listing operation of directory contents) takes place on the data channel. Each transfer initiates the creation of a new data path. In other words, the data channel is created and destroyed for each transfer. In addition, the data transfers that take place within a given FTP session are strictly sequential. A new transfer connection can not be started before the previous one has ended. This is in contrast to HTTP where the transfers within a given session may overlap in time.

\subsection{Session Structure}

A FTP session is considered to begin with the user authentication phase when userid (real or anonymous) and password are transmitted to server. The server validates the user on the client side and signals the start of a valid session on the control channel. The sequence of FTP commands and responses is given by RFC959 [23].

For a given session, a data transfer is started by user commands such as GET, PUT, or a directory listing, which are exchanged as RETR, STOR, or LIST protocol messages on the control connection. For modeling purposes, the LIST operation is considered to be equivalent to a GET operation. This is because the difference between the two is only in the nature of the content. In case of GET, the result is the real file content, whereas for LIST the result is the output of a program to list the contents of the directory.

The data channel is created on an on demand basis, between port 20 at server and an ephemeral port at client. Initiation of data channel connection is also from server to client. This is known as the active mode data transfer. Modern implementations of FTP services (e.g., FTP services accessed via browsers) are increasingly employing the passive mode of data transfer. Here, data channel establishment is initiated by the client. In addition, the server port is not 20 but an ephemeral port. In either case, the core issue is to coordinate the session so that the data channel setup (and teardown) can be performed smoothly. This is done via PORT messages. For active mode FTP sessions, the client opens a TCP socket and listens on one of its ephemeral ports. Using the control channel (already open), it then communicates the port number to server by using the PORT message. The server then completes the data connection by opening a connection from its port 20 to the designated port on the client side. In the case of passive mode data transfer, a role reversal takes place. The server opens a TCP socket on one of its ephemeral ports (and does a listen) and transmits the port number using a PORT message on the control channel. The client then does the active connect to create the data path. Using the established data channel, the 
contents of the requested file is transmitted. At the end of transfer, data connection is closed by the sending entity (client in case of PUT and server in case of GET). For the entire duration of data transfer, there are no message exchanges on the control connection. The end of session is marked by a QUIT command from the client.

\subsection{FTP Characteristics}

In order to build a realistic simulation model for the file transfer application, the following elements of FTP sessions have been investigated:

- FTP session arrivals. This statistics shows the frequency of occurrence of FTP sessions, indicating the behavior of individual users.

- Number of transactions within a session. Once a session is established, the user may perform one or more basic operations of type GET, PUT or LIST. We ignore, for simulation purposes, multi-transaction operations like MGET and MPUT. Each of the basic operations constitute one transaction within the session. Assuming that there are $N$ transactions within a session and the transaction durations are independent and identically distributed, then the distribution of session durations is likely to be the same as the distribution of the transaction durations convolved $N$ times. Usually, the users transfer a file and then spend sometime thinking about the next operation. This is similar to the active OFF times in HTTP transfers.

- Protocol message timings. The FTP session establishment, teardown, and the signalling exchanges between the client and the server is performed by passing protocol messages forth and back between the two entities. These messages are short messages transmitted as single packets (easily fitting into one TCP segment) and follow a strict request-response paradigm. Sending the protocol messages or the responses thereof constitutes an ON period on the control channel. On the other hand, the time gap between two consecutive protocol messages or two consecutive protocol responses gives rise to an OFF period, which also depends upon the network conditions in the end-to-end path.

- Content sizes. The content size distribution is the major contributor towards the transaction durations. The contents are drawn from the file systems. With the growing evidence of heavy-tailed nature of sizes of files, it is expected that the content sizes of FTP transmissions will be also heavy-tailed. The other factor that comes into play during the content transfers is the link speed.

- GET vs PUT probability. The FTP service allows for bidirectional data transfers. Depending on the relative frequency of GET and PUT operations, a FTP session may result in net data transfer from the server to the client or vice versa. This is a statistic that represents the user behavior.

An important observation is that, due to limited amount of collected data traces for FTP, our results can not be generalized to capture, e.g., daily variations of access, or human timescales, or geographical aspects.

\subsection{Traffic Analysis}

The analysis of FTP application traffic on both server and client side traces was performed to arrive at realistic models that can be used as input parameters to the simulation model of FTP client-server interaction. The server side traces were in the form of server access logs. The client side traces were generated in the form of Ethernet packet dumps. The analysis of these traces has yielded the following specific characteristics for the FTP service:

- The arrival process of FTP sessions has been found to be well modeled by Poisson processes. Alternatively, the inter-arrival time of FTP sessions can be modeled as an Exponential distribution. Analysis of two server access logs from SUNET shows a mean inter-arrival rate of 1.01 and 1.35 seconds (table 5). The maximum inter-arrival time was recorded as 25 seconds in the February access log and 15 seconds for the March access log. This indicates a lot of activity at the server. We mention that both access logs were taken starting around 22:00 hrs in the night and continued beyond midnight. The server being a popular anonymous FTP site, this is as per expectation. To preserve confidentiality, the server access logs were pre-processed at the Uppsala University to mask out the real userid and hostnames on the client side. In the absence of such information, it is difficult to ascertain whether the accesses were from the Scandinavian region or from other geographical regions where it was still normal working hours. Given the tremendous popularity of Internet all over the world, it is perhaps safe to attribute a major portion of the midnight traffic to regions outside Scandinavia. On the contrary, the server access logs at IIT Bombay show very low level activity. On the average, the server at IIT Bombay records about 5 FTP sessions per day. Consequently, the statistics for the session arrivals were not computed for IIT Bombay server. 


\begin{tabular}{|c|c|c|}
\hline Stat Name & Dataset Identifier & $\begin{array}{c}\text { Min, Median, Mean, Max } \\
\text { (in seconds) }\end{array}$ \\
\hline \hline Session Arrival & SUNET, February & $0,1,1.014,25$ \\
\cline { 2 - 3 } & SUNET March & $0,1.0,1.35,15$ \\
\hline Session Duration & SUNET, February & $0,41,304,5565$ \\
\cline { 2 - 3 } & SUNET, March & $0,95,452,9904$ \\
\cline { 2 - 3 } & IIT Bombay, February & $3,66,705,20580$ \\
\cline { 2 - 3 } & IIT Bombay, March & $7,67,743,21210$ \\
\hline Transfer Duration & SUNET, February & $1,1,88.16,5444$ \\
\cline { 2 - 3 } & SUNET, March & $1,8,291,8684$ \\
\cline { 2 - 3 } & IIT Bombay, February & $1,1,12.5,19620$ \\
\cline { 2 - 3 } & IIT Bombay, March & $1,1,69,20710$ \\
\hline
\end{tabular}

Table 5: Statistics for FTP sessions

- The client side packet traces were analyzed to characterize the end-to-end protocol message exchanges (table 6). Such statistics can only be gathered by collecting packet dumps. As mentioned above, the client side accesses at BIT Karlskrona and at IIT Bombay were very low. The message size statistics have been computed by studying limited available data. In some cases, the nature of the messages has been studied and suitable models have been derived by using logical reasoning about the structure of the messages and also by using plausible estimates for the structural elements. For example, the initial response from the FTP server (on establishment of control connection from the client end) has 3 components. The first is the response code 220. The second component is the server name. Depending on the implementation and the configuration of the software, it may just be the host name or, alternatively, be a fully qualified domain name. Since the data available was limited, the variability in this component has been assumed to be in the range of 10-40 bytes. The third component of the initial server message is the FTP server software version id. Again, this has been assumed to be in the range of 10-50 characters. As a result, the total initial message size from the server has been approximately represented as a Uniformly distributed random variable in the range of 30-100 bytes.

\begin{tabular}{|c|c|c|}
\hline Message Type & $\begin{array}{c}\text { Distribution } \\
\text { Model }\end{array}$ & $\begin{array}{c}\text { Model Parameters } \\
\text { (in bytes) }\end{array}$ \\
\hline \hline Initial Server Response & Uniform & $\min =30, \mathrm{max}=100$ \\
\hline User id & Uniform & $\min =5, \mathrm{max}=10$ \\
\hline User Passwd & Uniform & $\min =5, \mathrm{max}=30$ \\
\hline Login Response & Constant & 25 \\
\hline CWD command & Uniform & $\min =5, \max =40$ \\
\hline CWD Response & Constant & 35 \\
\hline LIST command & Constant & 4 \\
\hline PORT command & Constant & 30 \\
\hline PORT Response & Constant & 65 \\
\hline GET/PUT command & Uniform & $10-50$ \\
\hline GET/PUT Response & Uniform & $40-90$ \\
\hline
\end{tabular}

Table 6: Messages exchanged on FTP control connection and their models

- All data transfers seen at the SUNET server were anonymous accesses. As a result, there were $100 \%$ GET type transfers. The server access logs for February 2000 at IIT Bombay shows 98.3\% GET and 1.7\% PUT type transfers and for March 2000 it shows 38\% GET and 62\% PUT type transfers.

- The number of transactions likely to occur during a FTP session for the anonymous FTP sessions at SUNET and at IIT Bombay are shown in figure 15. During the analysis, it turned out that a large number of sessions had zero transfers. This behavior, i.e., opening a FTP session and not performing any operation (e.g., GET, PUT, or LIST), is disturbing. However, it may be recalled that the server logs do not show the LIST operations. It is possible that a large fraction of zero-transaction sessions may actually have performed LIST operations (and thus had really some GET type transactions). Also, there were a few sessions in each dataset having more than one hundred transactions during a session. The investigation of such high transaction FTP sessions revealed that the users were downloading, e.g., the entire tree of RFC documents or installing Linux onto the client machines. Presently such type of usage of the FTP service is pretty common. For example, FTP sessions are likely to occur close on the heels of each release of RedHat Linux with the users trying to upgrade their installations. Figure 15 shows the CCDF plot for all datasets using a log-log scale. The linear declining behavior indicates that Pareto distribution can be used to model this phenomena. However, the AD test for the Pareto distribution does not show any 
significance. This indicates that the model is not valid from a statistical point of view. On the other hand, the large range of values (four orders of magnitude) seen in the observations suggests that the underlying distribution is a heavy-tailed distribution such as Pareto. Another point is that we visually note the roughly parallel behavior of the curves. By using a least square line passing through the points, the value of $\alpha$ parameter was roughly estimated to be 1.3 . The scale parameter $\beta$ in all datasets has a value of 1 . The statistics for different datasets are shown in the table 7 . The minimum number of transactions that occurred during the recorded sessions is zero. This value can not be used as the scale parameter $\beta$ in the Pareto model of the number of transactions because a $\beta$ value of zero will always generate a number of zero transactions. In order to resolve this, only the sessions with non-zero transactions are considered for modeling. Accordingly, for each dataset there are two rows shown in the table 7 . The first row gives the values where the zero transaction sessions are retained and the second row gives the values where the zero transaction sessions are discarded. The differences in the mean values can be noted. However, the plots with and without zero transaction sessions do not show any appreciable change in the linear decay structure in CCDF plots.
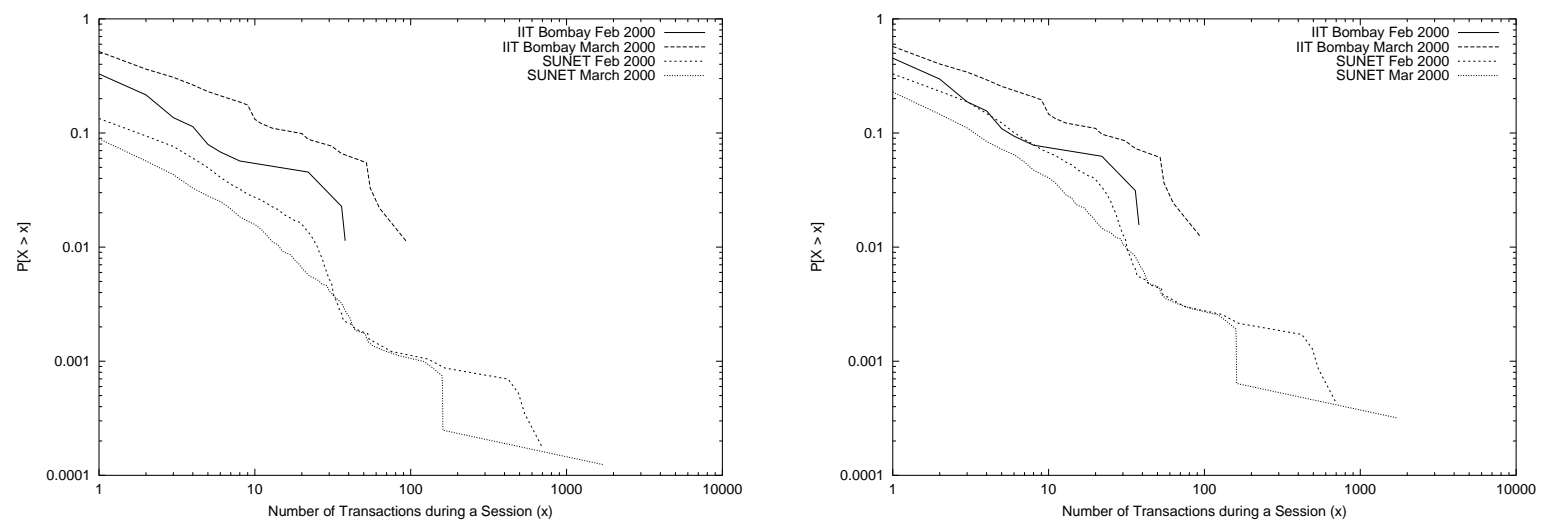

Figure 15: CCDF of the number of transactions occurring within a FTP session: with zero transaction sessions (left) and without zero transaction sessions (right)

\begin{tabular}{|c|c|c|}
\hline Dataset & $\begin{array}{c}\text { Zero } \\
\text { transactions } \\
\text { (percentage) }\end{array}$ & $\begin{array}{c}\text { Min, Median, Mean, Max } \\
\text { (counts) }\end{array}$ \\
\hline \hline SUNET Feb & 59 & $0,0,2.25,3150$ \\
\cline { 2 - 3 } & - & $1,1,5.55,3150$ \\
\hline SUNET March & 61 & $0,0,1.52,2342$ \\
\cline { 2 - 3 } & - & $1,1,3.91,2342$ \\
\hline IIT Bombay Feb & 28 & $0,1,37.4,3048$ \\
\cline { 2 - 3 } & - & $1,1,51.45,3048$ \\
\hline IIT Bombay March & 10 & $0,2,8.22,108$ \\
\cline { 2 - 3 } & - & $1,2,9.12,108$ \\
\hline
\end{tabular}

Table 7: Statistics for number of transactions within FTP sessions

- The files transferred during FTP sessions constitute the actual payload. The CCDF of the file sizes are shown in figures 16 and 17. In the case of IIT Bombay transfers, the file uploads and downloads have been shown separately. In most cases, the heavy-tailed nature of the file sizes is observed. The number of observations collected from IIT Bombay is small and is spread over long periods (one month each). In such cases, the data collected tends to be non-homogeneous. It does not show a clear structure and presents difficulties during modeling. The observations collected from SUNET show better behavior. The file sizes have been modeled using a combination of Lognormal and Pareto distributions. The results of the modeling process along with the parameters of the distributions are shown in table 8 . The February dataset from SUNET shows good results when AD test is applied for subsamples of 500 for the Lognormal body portion and subsamples of 100 for the Pareto tail. On the contrary, for the March dataset from SUNET, the Lognormal body shows statistical significance only with subsamples of size 100 in the Lognormal body and the Pareto tail does not show any significance even at subsamples of size 50. This may be attributed to the fact that in the March dataset from SUNET, there seems to be a third random process starting around file sizes greater than $10^{8}$. In all datasets it was generally observed that there were some ranges in the file sizes where there were no observations recorded (giving rise to gaps). Nevertheless, the large spread in the 
values of observations and the linear decay behavior in the tail indicates that the Pareto distribution is the most likely candidate. The IIT Bombay March datasets show some significance values for the Lognormal body and Pareto tail but the fitting is poor and therefore not shown in the table.

\begin{tabular}{|c|c|c|c|c|c|c|}
\hline Dataset & $\begin{array}{c}\text { Lognormal } \\
\text { Parameters } \\
(\mu, \sigma)\end{array}$ & $\begin{array}{c}\text { Sub } \\
\text { sample } \\
\text { size }\end{array}$ & $\begin{array}{c}\text { Pareto } \\
\text { Parameters } \\
(\alpha, \beta)\end{array}$ & $\begin{array}{c}\text { Sub } \\
\text { sample } \\
\text { size }\end{array}$ & $\begin{array}{c}\text { Tail } \\
\text { mass }\end{array}$ & $\begin{array}{c}\lambda^{2} \\
\text { value }\end{array}$ \\
\hline \hline SUNET Feb & $10.72,2.39$ & 500 & $0.969,71678$ & 200 & 0.09 & 0.12 \\
\hline SUNET March & $11.42,2.57$ & 100 & $1.08,239334$ & - & 0.10 & 0.25 \\
\hline
\end{tabular}

Table 8: Results of fitting models to FTP file sizes
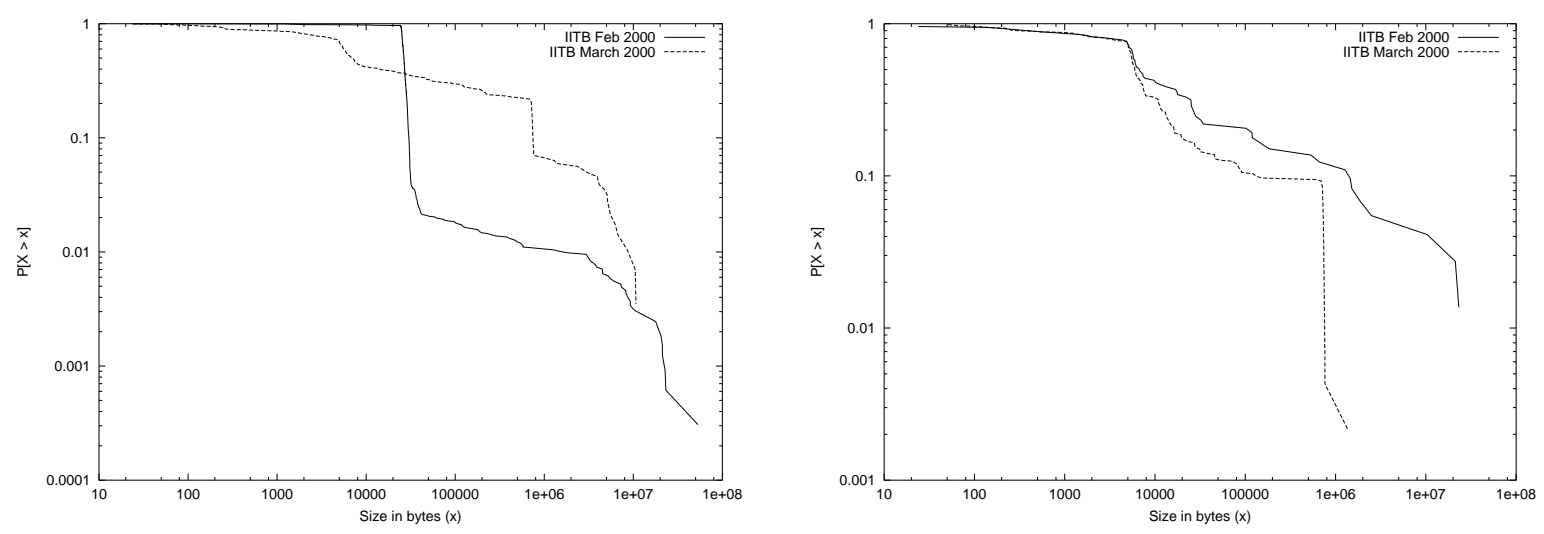

Figure 16: CCDF of the transferred file sizes at the IIT Bombay FTP server : downloads (left), uploads (right)

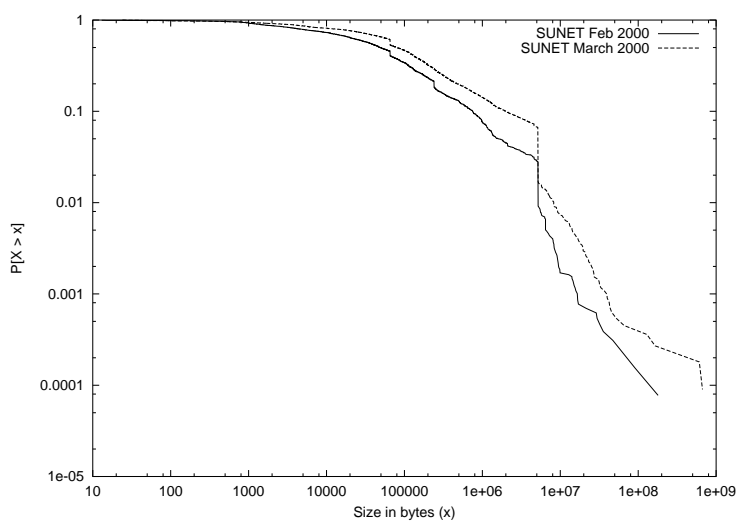

Figure 17: CCDF of transferred file sizes at the SUNET FTP server (anonymous downloads)

\section{Summary}

A characterization study of traffic collected at clients and servers for the "classical" Internet applications SMTP, HTTP, and FTP has been presented. Detailed results have been reported on measuring, modeling and analysis of traffic collected from different classes of sites. The modeling activity focuses on how the users typically access the applications, kind of demands they generate as well as how the TCP/IP protocol stack handle these demands. To this end, real network traffic environments have been monitored to collect real packet traces and these traces have been used to characterize the applications. Packet traffic has been collected from different environments such as university networks and commercial Frame Relay networks. Statistical models have been developed for diverse parameters of applications. All three applications possess a session oriented structure. Within each session, a number of transactions are performed, and this number has been also modeled. 


\section{References}

[1] Arlitt M. and Williamson C., Internet Web Servers: Workload Characterization and Performance Implications, IEEE/ACM Transactions on Networking, Vol 5, No 5, October 1997.

[2] Barford P. and Crovella M., Generating Representative Web Workloads for Network and Server Performance Evaluation, ACM SIGMETRICS 1998.

[3] Barford P., Modeling, Measurement and Performance of World Wide Web Transactions, PhD thesis, Department of Computer Science, Boston University Graduate School of Arts and Sciences, USA, 2001.

[4] Berners-Lee R. et al., Hytertext Transfer Protocol-HTTP/1.0, RFC 1945, May 1996.

[5] Cooper I. and Dilley J., Known HTTP Proxy/Caching Problems, RFC 3143, June 2001.

[6] Crovella M.E. and Taqqu M.S., Estimating the Heavy Tail Index from Scaling Properties, Methodology and Computing in Applied Probability, Vol 1, No. 1, 1999.

[7] D’Agostino R.B. and Stephens M.A., Goodness-of-Fit Techniques, Marcel Dekker Inc., 1986.

[8] Danzig P.B., Jamin S., Caceres R., Mitzel D. and Estrin D., An Artificial Workload Model of a TCP/IP Internetwork, Journal of Internetworking: Research and Experience, Vol. 3, pages 1-26, 1992.

[9] Fielding R. et al., Hypertext Transfer Protocol - HTTP/1.1, RFC 2616, June 1999.

[10] Heidemann K., Obraczka K., and Touch J., Modeling the Performance of HTTP Over Several Transport Protocols, IEEE/ACM Transactions on Networking, Vol 5, No 5, October 1997.

[11] Jena A.K., Modeling and Analysis of Internet Applications, Licentiate Thesis, University of Lund, Sweden, 2000.

[12] Jena A.K. and Popescu A., Traffic Engineering for Internet Applications, The Conference on Internet Performance and QoS, ITCom2001, Denver, USA, August 2001.

[13] Johnson N., Kotz S., and Balakrishnan N., Continuous Univariate Distributions, Vol. 1, Second Edition, John Wiley \& Sons, 1994.

[14] Krishnamurthy B. and Rexford J., Web Protocols and Practice, Addison-Wesley, 2001.

[15] Kurien T.V., correspondence with T. V. Kurien (Niksun Inc.), 1998.

[16] Mah B.A., An Empirical Model of HTTP Network Traffic, INFOCOM 1997, Kobe, Japan, April 1997.

[17] NIKSUN NetVCR ${ }^{T M}$, http://niksun.com/products/netvcr.html

[18] Ostermann S., TCPTRACE: A TCP connection analysis tool http://jarok.cs.ohiou.edu/software/tcptrace/tcptrace.html.

[19] Paxson V., Empirically-Derived Analytic Models of Wide-Area TCP Connections, IEEE/ACM Transactions on Networking, Vol. 2, No. 4, August 1994.

[20] Paxson V. and Floyd S., Wide-Area Traffic: The Failure of Poisson Modeling, IEEE/ACM Transactions on Networking, Vol. 3, 1995.

[21] Postel J.B., Simple Mail Transfer Protocol, RFC 821, August 1982.

[22] Crocker D.H., Standard for the Format of ARPA Internet Text Messages, RFC 822, August 1982.

[23] Postel J.B. and Reynolds J.K., File Transfer Protocol, RFC 959, October 1985.

[24] Resnick S.I., Heavy Tail Modeling and Teletraffic Data, The Annals of Statistics, 25(5), 1997.

[25] Willinger W., Paxson V. and Taqqu M.S., Self-Similarity and Heavy-tails: Structural Modeling of Network Traffic, A Practical Guide to Heavy Tails, Birkhäuser, Boston, 1998.

[26] Xanthakis S., GetWeb version 2.7.2, January 1999, http://www.enfin.com/getweb/ 


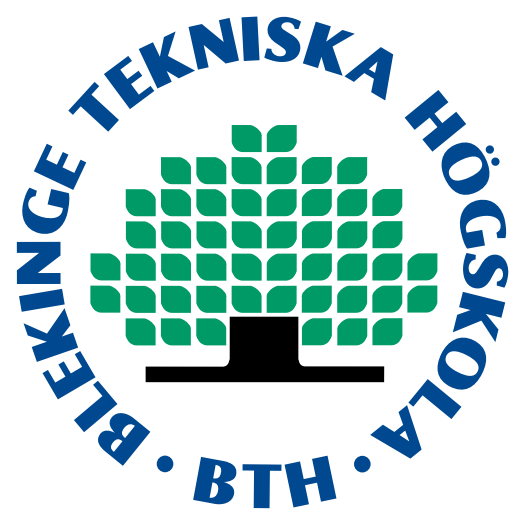

Modeling and Evaluation of Internet Applications by Ajit K. Jena, Adrian Popescu and Arne A. Nilsson

ISSN 1103-1581

ISRN BTH-RES--08/02--SE

Copyright () 2002 by individual authors

All rights reserved

Printed by Kaserntryckeriet AB, Karlskrona 2002 